\title{
A piezo-based bearing for the active structural acoustic control of rotating machinery
}

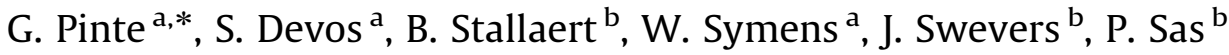 \\ a Flanders' MECHATRONICS Technology Centre, Celestijnenlaan 300D, B-3001 Leuven, Belgium \\ ${ }^{\mathrm{b}}$ Katholieke Universiteit Leuven, Department of Mechanical Engineering, Celestijnenlaan 300B, B-3001 Leuven, Belgium
}

\section{A R T I C L E I N F O}

\section{Article history:}

Received 28 July 2009

Received in revised form

26 October 2009

Accepted 28 October 2009

Handling Editor: J. Lam

Available online 24 November 2009

\begin{abstract}
A B S T R A C T
This paper presents an active bearing for reducing the radiated structure borne noise of rotating machinery. This modular bearing uses piezostacks to generate secondary forces in the vibration transmission path of the controlled structure. The bearing is tested on an experimental test bed comprising a rotating shaft, which is mounted in a frame. Noise is radiated by a plate that is attached to the frame. To evaluate different control approaches, a simplified model of the set-up was made. Based on the simulation results, a combination of feedback and repetitive control is implemented, using a force and acceleration measurement. This way, a noise reduction of more than $10 \mathrm{~dB}$ is achieved at the most important resonance frequencies of the system below $1 \mathrm{kHz}$.
\end{abstract}

(c) 2009 Elsevier Ltd. All rights reserved.

\section{Introduction}

Modern society is characterized by an increasing attention for the environmental impact of newly developed machinery. One of the environmental criteria that is gaining importance is the noise emission level produced by a new machine since the legal regulations more and more restrict the allowable noise levels. Moreover, the noise level of a machine is also becoming an important competitive quality of the product. To cope with these emerging demands, new techniques to reduce the noise level of machines have to be investigated. Whereas currently mostly passive techniques such as sound absorption or isolation are used, active control techniques will be increasingly applied to design more silent machinery. In contrast with the traditional passive noise control measures, which are effective at high frequencies but become bulky and inefficient at lower frequencies, active control is particularly suitable for the cancelation of low frequency noise.

A popular active control technique for the reduction of structure borne noise is active structural acoustic control (ASAC) $[1,2]$. An ASAC system uses structural actuators to intervene in the vibrations of a mechanical system with the objective of attenuating the radiated noise level. Depending on the location where the active element operates in the vibration propagation path, two ASAC approaches can be distinguished. On the one hand, the vibro-acoustic response of the noise radiating surfaces can be controlled by active means directly attached to these surfaces [3,4]. On the other hand, active techniques can also be applied to block forces in the vibration transmission paths towards the noise radiating surfaces, when it is impossible or impractical to control the vibro-acoustic response of all the noise radiating surfaces [5]. In this article, the second approach will be followed by the development of a modular active piezo-based bearing for rotating machinery.

\footnotetext{
* Corresponding author. Tel.: +32 16328035 .

E-mail address: gregory.pinte@fmtc.be (G. Pinte).
} 
Excessive noise radiation from a structure housing a rotating device is a common problem in many industrial applications such as gearboxes [6], compressors, etc. The disturbance noise in these applications is often generated by forces on the rotating device e.g. gear mesh forces or air compression forces, which are transmitted through the bearings to the noise radiating machine frame. Previous research in this domain mainly focussed on the active noise and vibration reduction of gearboxes. Guan et al. [7] have compared four different actuation concepts for the active suppression of gearbox housing vibrations. Criteria which have been taken into account in this comparison, are ease of implementation, actuation effort and control robustness. The conclusion of this numerical study is that executing translational forces on the vibration transmission path is favorable compared to controlling the torsional degree of freedom of the gear or the shaft. Typical locations to apply these translational forces are on the one hand on the existing gearbox bearings [8] and on the other hand through an additional bearing on the shaft [9,10]. Guan et al. [7] state that the introduction of an additional bearing can be beneficial to damp the shaft bending modes.

While the number of applications of active systems used to suppress the noise from rotating machinery is still restricted, similar active systems have been used extensively in the field of rotor dynamics. In rotor dynamic applications, the objective is to restrict the vibrations of the flexible rotor. Especially when a rotating machine passes through its critical speeds, the rotor vibrations are excessive, which limits the system's accuracy and sometimes even leads to failure of the machine. Palazzolo et al. [11] have analyzed the potential of a piezoelectric actuator based active vibration control system for these rotor bearing systems and applied it successfully on a jet engine. Next to piezoelectric actuation techniques, also other actuation techniques have been used for rotor dynamics control such as active magnetic bearings [12,13], actuators based on shape memory alloy [14,15], etc.

In contrast with the rotor dynamics applications, the objective in the presented work is not to decrease the rotor vibrations but to limit the radiated noise by limiting the force transfer from the shaft to the housing. Since the disturbance forces are transmitted through the bearings to the machine frame, it was decided in this research to incorporate the active elements in the bearings. This approach has already been followed in the field of rotor dynamics [16]. However, the tackled frequency range in rotor dynamics control is much lower $(<100 \mathrm{~Hz})$ than the frequency range of interest in noise control, which is studied in this paper $(<1 \mathrm{kHz})$.

After the design of an actuator and the placement of this actuator at the appropriate location in the controlled application, the last important step in the design of an active system is the development of a suitable control algorithm [17]. Basically, the various control algorithms, which are mainly used in ASAC, can be classified into two categories: feedforward and feedback algorithms. The most popular control algorithm in ASAC systems for rotating machinery is the filtered-X least mean squares (FxLMS) algorithm, which is an adaptive feedforward control technique. A good overview of the working principle of this algorithm can be found in [18]. The FxLMS algorithm is based on the availability of a reference signal, like the fundamental frequency of the disturbing noise. In applications where the disturbance is periodic (e.g. rotating machinery) this can usually be provided by a tacho signal. This reference is filtered through a control filter to calculate the driving signal for the secondary actuator. In order to cope with the changes in the controlled system (non-stationary disturbance, varying environmental conditions, etc.), the control configuration is augmented with additional sensors, installed at the locations where noise/vibration reduction is required. Based on these additional sensor signals, the control filter is adjusted to the system's variation. Recently, adaptive repetitive control is presented as a valuable alternative for the conventional FxLMS algorithm [19]. This latter algorithm, which will be applied for the control of the bearing, will be described in detail in Section 4.2.

Next to feedforward control algorithms, feedback algorithms are also employed in ASAC to reject a disturbance signal. Stability and robustness are the crucial elements in the design of feedback controllers. Two main approaches can be distinguished for the design of feedback controllers: the traditional transfer function based approach and the modern model-based approach. In the first approach [20,21], the controllers are developed directly based on the transfer function of the controlled plant, using graphical techniques such as Nyquist, Bode and root-locus methods. The most important drawback of this transfer function based approach is the fact that it becomes very involved in the case of multiple input multiple output (MIMO) systems. Furthermore, this traditional approach is not able to optimize certain desired performance criteria. In the second approach, state-space models are used to describe the controlled system behavior. Modern design methods [22] are then applied on these identified models to define appropriate feedback control laws such that certain performance and stability criteria are fulfilled. Examples of these modern design methods are the LQR, LQG $[23,24]$ and $H_{\text {inf }}[25]$ techniques. The applicability of these modern design methods is often limited in ANC and ASAC applications since it is complicated to build a reliable state-space model of the considered acoustic system due to the significant time delays and the high number of modes in the controlled frequency range. Therefore in this paper the feedback controllers will be developed according to the transfer function based approach.

This paper presents the complete development (design, control and analysis) of an active bearing for reducing the radiated structure borne noise of rotating machinery. In the following section of this paper, the design of this active bearing will be presented. This section will also describe the experimental test bed, which is used to evaluate the effectiveness of the bearing. In the third section, a simplified model of this test bed will be derived, on which the theoretical possibilities of different control strategies can be compared. The results of the implemented control strategies will be discussed in the fourth and the fifth section. The fourth section will present a controller based on the transmitted force through the bearing. To cope with the limitations of this controller, a second controller, which uses the frame acceleration as the error signal, will be discussed in Section 5. Finally, the obtained results will be summarized in the conclusions. 


\section{Design of the piezo-based bearing}

The idea behind the design of an active bearing is to affect the force transmission from the shaft to the machine frame by the introduction of secondary forces in the bearing. The objective is to control these applied forces based on a measurement of the transmitted force through the bearing. For the practical implementation of this concept collocated piezoelectric actuators and sensors are integrated in a ring-shaped module that is mounted around a regular existing bearing. A detailed view of such a sensor-actuator pair is shown in Fig. 1. The piezosensor and -actuator are mounted in series, such that the complete generated actuator force is transmitted through the sensor. The cross-section of the selected piezosensor-actuator pair amounts to $25 \mathrm{~mm}^{2}$ and its length amounts to $16 \mathrm{~mm}$. Although the piezoactuator has a sufficient stroke to compensate the disturbances on the set-up (see further), it is acknowledged that in stiff industrial applications, which are excited by larger forces, longer piezoactuators with larger sections should be used to generate the required strokes.

To be able to generate forces in all radial directions, a horizontal and vertical sensor-actuator pair is introduced in the developed ring-shaped module. A drawing and a picture of this module are shown in Figs. 2(a) and (b). This active element provides the interface between the frame and an existing bearing. The piezoactuators and -sensors are preloaded by a spring, such that they are capable of applying, respectively, measuring radial bi-directional (push/pull) forces. For each piezoactuator, the necessary voltage over the electrodes to generate a required force is delivered by a power amplifier. The electrodes of each piezoelectric sensor are connected to the input of a charge amplifier. This way, the force through the piezosensor, which is proportional to the charge displacement, can be measured.

The mechanical structure of the developed active element is designed such that both sensor-actuator pairs are decoupled, all force is transmitted through the piezos and shear stresses in the piezos are avoided. Decoupling is an important element in the mechanical design since it can considerably simplify the control design afterwards. Perfect mechanical decoupling implies that the control and disturbing forces of the actuator in one direction are only sensed by the collocated sensor and not by the sensor in the perpendicular direction. In that case, two independent single input single output (SISO) controllers can be applied for the control of both collocated systems. In practice, some coupling is inevitable and the amount of coupling will determine whether SISO control is still possible or whether a full multiple input multiple output (MIMO) control design must be applied. One of the possible causes for coupling between the horizontal and vertical direction are the gyroscopic coupling terms, which show up in the equations of the shaft motion. Gyroscopic effects become more pronounced as the rotational speed increases and as the ratio between the polar and the transverse moment of inertia of the shaft becomes larger [26,27]. In this set-up, the gyroscopic coupling will be limited, since the applied rotational speeds are limited and the ratio of the polar and transverse moments of inertia of the shaft with a diameter of $50 \mathrm{~mm}$ and a length of approximately $300 \mathrm{~mm}$ is relatively small. Consequently, two SISO controllers will be used for the control of the piezoelectric bearing in this case. In gearboxes with short shafts and large gears, the coupling can become higher such that a more advanced MIMO controller can be more appropriate than two SISO controllers.

A second important element in the mechanical design is the avoidance of secondary force transmission paths between the bearing and the frame. This way, the complete transmitted force passes through the piezos and can be measured accurately, which limits the complexity of the control design. The mechanical design should also avoid shear stresses in the piezostacks, which drastically reduce their life expectancy and can even cause immediate failure. Practically, all these specifications are achieved by using leaf springs and hinges that are flexible in one direction and stiff in the perpendicular

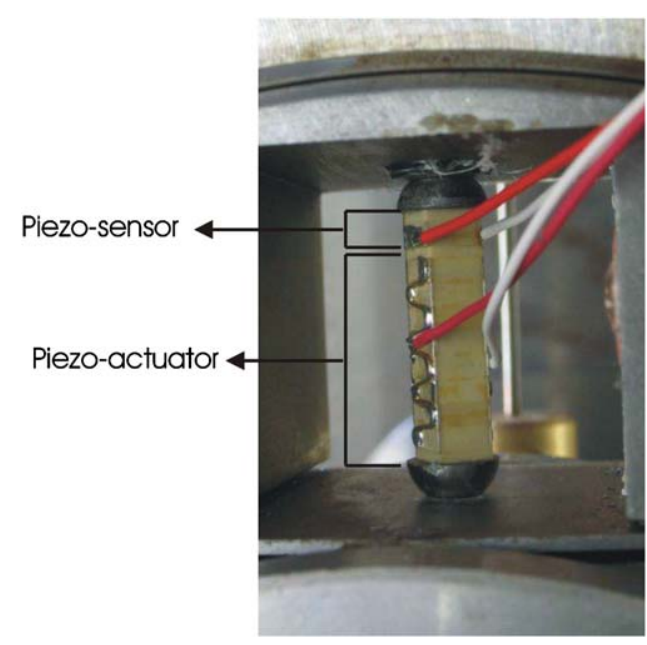

Fig. 1. The piezosensor and -actuator, mounted in series. 
(a)

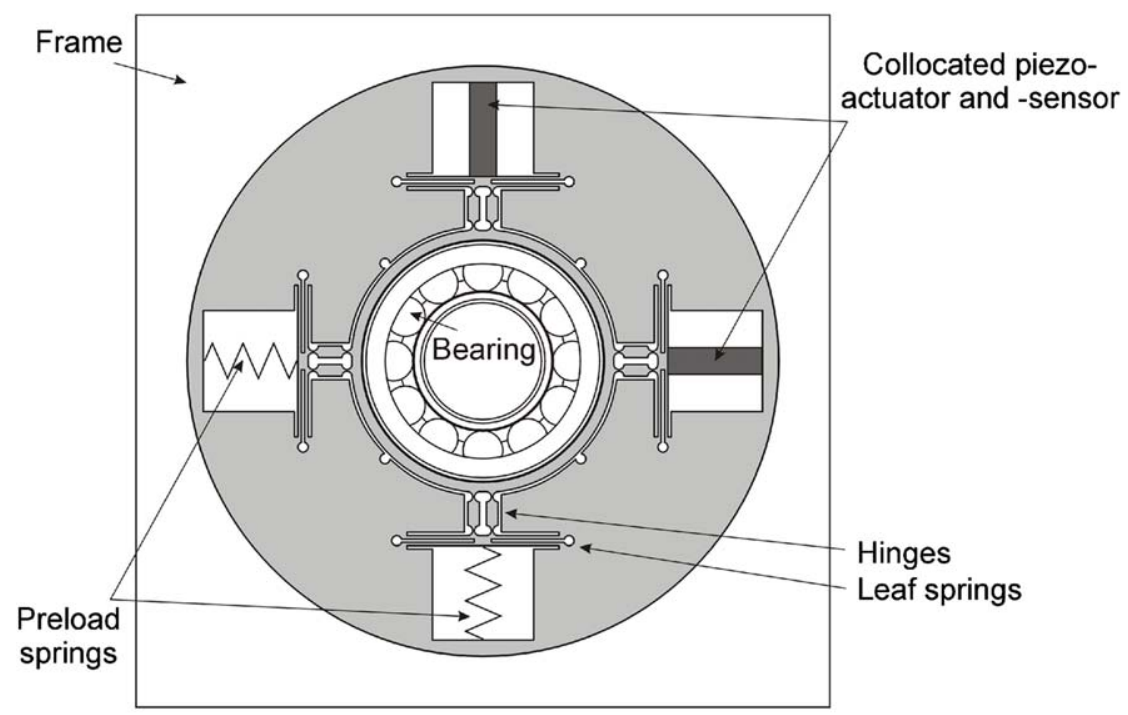

(b)

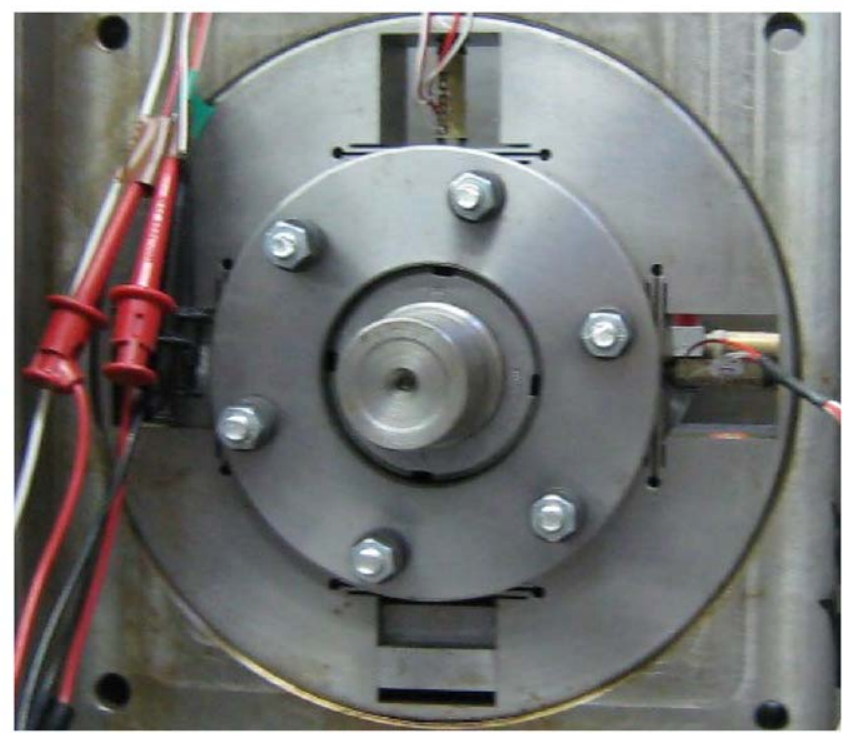

Fig. 2. The developed piezoactive bearing: (a) drawing and (b) picture.

direction This is illustrated in Fig. 3. Hinge 1 guarantees decoupling, while leaf spring 2 avoids shear stresses. The stiffnesses of both elements should be as low as possible as they introduce secondary force transmission paths. The leaf springs and hinges are manufactured by electrical discharge wiring.

In order to evaluate its practical performance, the modular active bearing is built in an experimental test bed (Fig. 4(a)). In this test bed, a motor drives a shaft, which is mounted with bearings in a frame. Noise is radiated by a plate that is attached to the frame. The dimensions of the different parts are chosen such that the test set-up has a representative dynamic and acoustic behavior for industrial rotating machinery such as compressors and gearboxes. Like these machines, the set-up exhibits in the frequency range of interest, up to $1 \mathrm{kHz}$, many plate resonances, some frame resonances and the resonance of the shaft on the bearing stiffnesses. Furthermore, this shaft resonance of the set-up, which is an important element in the dynamic and acoustic properties of rotating machinery as will be described further, appears in the same frequency range (around $700 \mathrm{~Hz}$ ) as in industrial compressors and gear boxes. The disturbance force on the shaft is induced by a shaker, which is attached to the shaft through a roller bearing. The shaft is supported by a cylindrical bearing at one side and a double angular contact ball bearing at the other side. At the latter side, the piezoactive ring-shaped module is placed around the bearing. The radial clearance has been avoided by a careful design with a spherical-roller bearing, in order not to compromise the stroke of the actuators. In the remainder of this article, the bearing with the piezoactive 


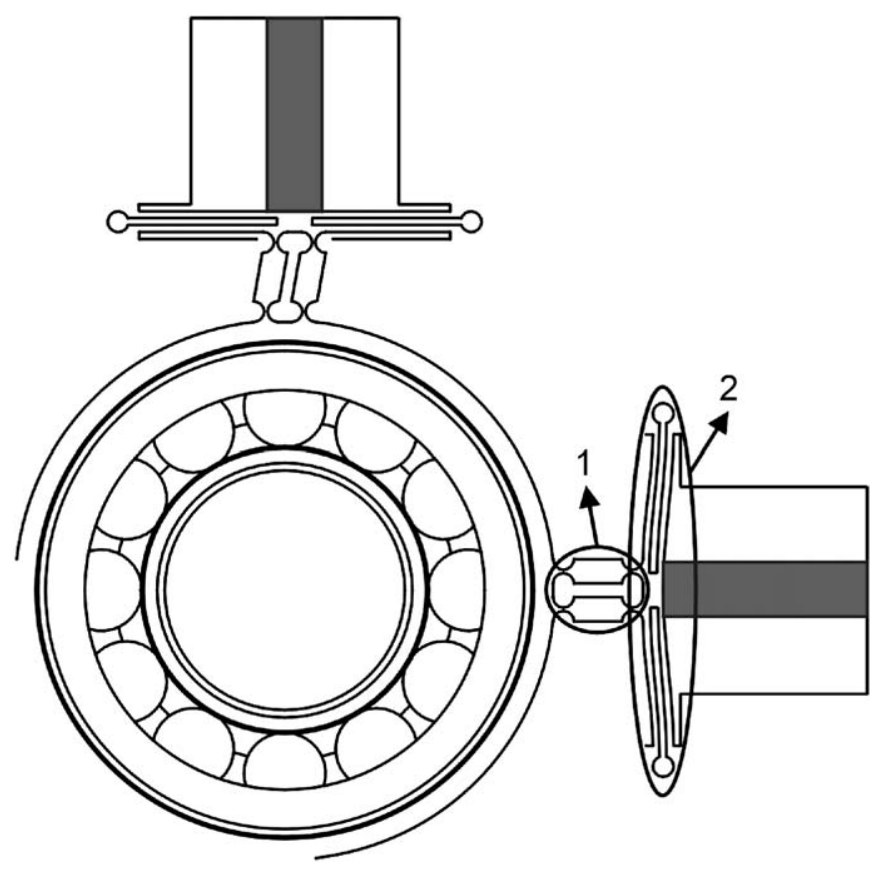

Fig. 3. The decoupling between the horizontal and vertical axes in the active system, achieved by using hinges (1) and leaf springs (2).

ring is called the active bearing, while the cylindrical bearing is called the passive bearing. Fig. 4(b) shows a photo of the test bed.

To evaluate the performance of the active bearing, different sensors are installed on and around the test bed. As mentioned above, the force through the two piezosensors is measured. Furthermore, a three-dimensional accelerometer and a one-dimensional accelerometer measure the vibrations, respectively, on the frame close to the active bearing and in one corner of the plate. A microphone registers the noise level in front of the plate at a distance of approximately $50 \mathrm{~cm}$. In some experiments, the motor is not driven and an additional three-dimensional accelerometer is mounted in the middle of the shaft to assess the effect of the ASAC strategy on the shaft vibrations. The signals from the piezosensors, the accelerometers and the microphone are recorded by a dSpace 1006 real time control board at a sampling frequency of $20 \mathrm{kHz}$. The piezoactuators are controlled at the same sampling frequency.

\section{Modeling and working principle}

In this section a simplified model of the test bed is presented in order to understand the working principle of the active bearing and to predict its theoretically achievable performance. The unidirectional lumped parameter model of the experimental test bed (for one direction) is shown in Fig. 5 . The shaft, represented by the mass $m_{s}$, is excited by a disturbing force $f_{d}$. As a consequence, a force is transmitted through the piezoactuator and -sensor from the shaft to the frame. This frame, represented by the mass $m_{f}$, is supported by compliant rubber mounts, modeled by the spring $k_{0}$. For the piezoactuator and -sensor (simplified) equivalent mechanical models [28] are used. $k_{a}$ and $k_{s}$ are the mechanical stiffnesses of the actuator and sensor, respectively, while the spring $k_{c}$ represents the contact stiffness introduced by the mounting of the piezoelements. The voltage to the electrodes of the piezoactuator is represented by an equivalent mechanical force $f_{a}$. For the piezoelectric sensor, the charge displacement is represented by the displacement $x_{s}$, which is proportional to the force acting on the sensor. Next to the primary force transmission through the piezoelements, there are also secondary force transmission paths from the shaft to the frame through the passive bearing, the hinges and the leaf springs. The spring $k_{p}$ comprises the stiffness of all these elements. In the practical set-up, the shaker force is applied on the shaft close to the active bearing to limit the effect of force transmission through the passive bearing. In the model in Fig. 5 only the first plate resonance $(210 \mathrm{~Hz})$ is represented by the introduction of the mass $m_{1}$ and the spring $k_{1}$. The resonance of the resulting mass-spring system corresponds to the first bending plate mode. The vibrations of $m_{1}$ can be considered as a good measure for the noise radiation of the set-up. A small amount of damping is introduced in the lumped parameter model by placing dampers $c_{0}, c_{1}$ and $c_{p}$ in parallel with the stiffnesses $k_{0}, k_{1}$ and $k_{p}$ such that the system's resonances have a damping ratio of approximately $0.1 \%$.

A comparison between the measured driving point transfer function between the shaker force on the shaft and the acceleration at the same point (Fig. 6(a)) and the transfer function obtained from the model (Fig. 6(b)) shows that the model captures the main elements of the set-up's dynamic behavior. The shaft antiresonance and resonance and the slope 
(a)

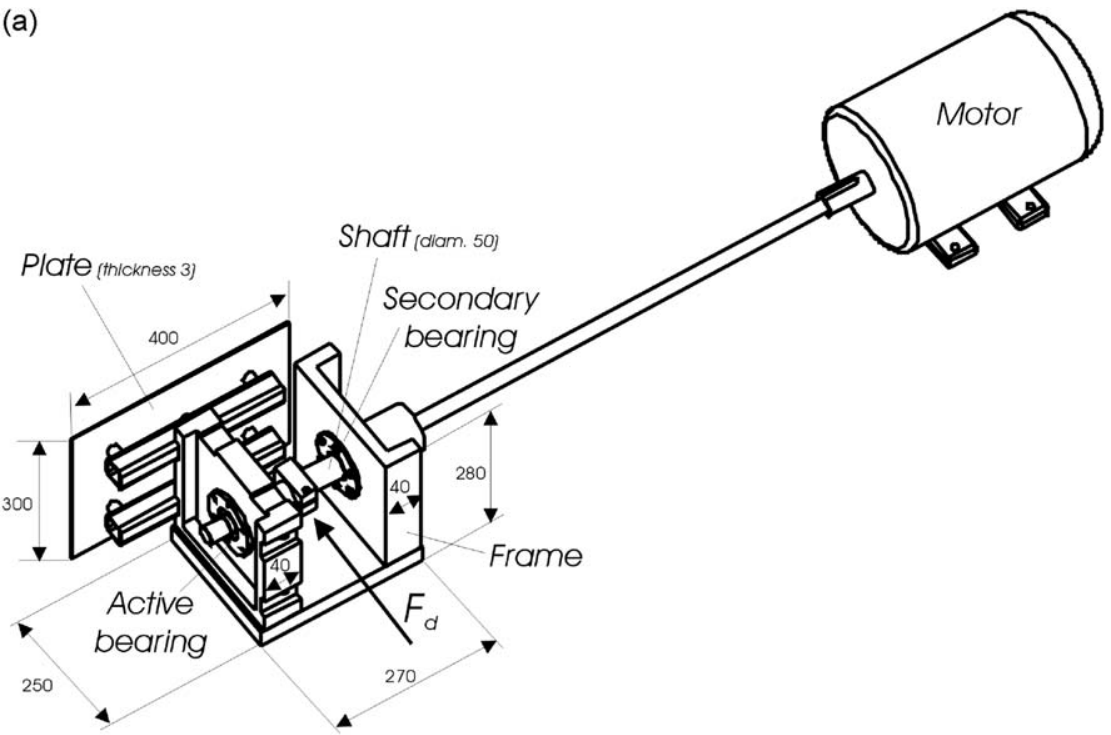

(b)

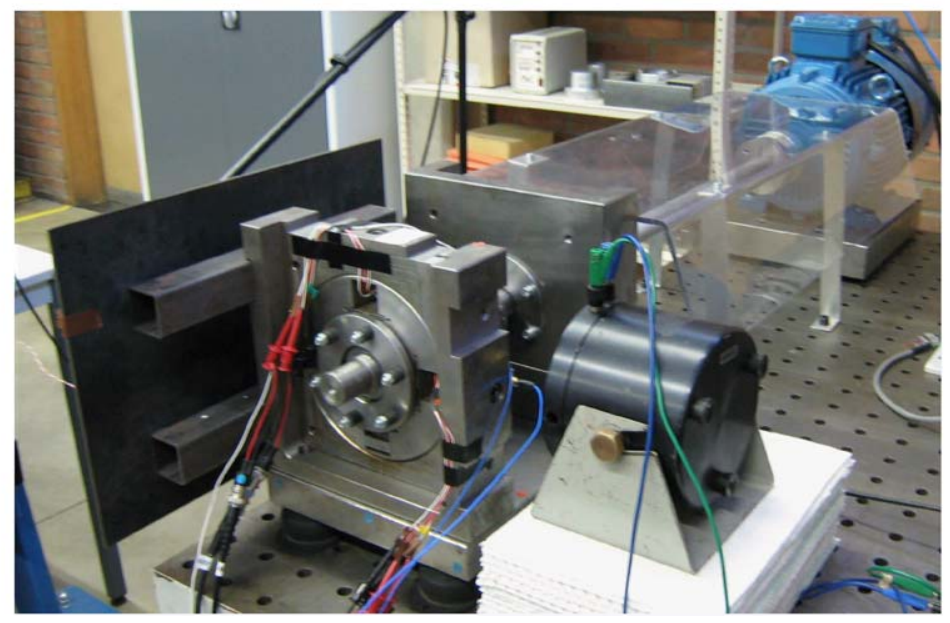

Fig. 4. The experimental set-up used to evaluate the performance of the piezoactive bearing: (a) a sketch (the dimensions are expressed in mm) and (b) a photo of the actual set-up.

of $+40 \mathrm{~dB}$ /decade in between, are present in both model and measurements. While in the measured frequency response function several plate modes arise as spikes on this slope, in the model, where the plate is only represented by one mass-spring system, only one mode shows up.

From this model, it is clear that the force measured by the piezoelectric sensor is not equal to the total transmitted force between the shaft and the frame, which consists of the measured force through the primary path and the force through the secondary path. Consequently, when the measured force between the shaft and the frame is controlled to zero, force can still be transmitted from the shaft to the frame through the secondary path. Due to this secondary force, the frame and the plate can still vibrate and noise radiation from the plate is still possible. On the other hand, since the disturbance force is acting on the shaft, the frame and the plate acceleration will be equal to zero when the transmitted force between the shaft and the frame is controlled to zero. From a theoretical point of view, ideal control of the transmitted force is therefore equivalent to ideal control of the frame acceleration, leading both to zero plate acceleration. This insight will be used in the next paragraph, when the sensor signals for the control experiments are chosen.

The transmitted force cannot be measured directly, but can be derived from the measured force and the input voltage to the piezoactuators according to the following equation:

$$
f_{\text {trans }}=\left(1+\frac{k_{p}}{k_{\text {prim }}}\right) \cdot f_{s}+\left(1+\frac{k_{p}}{k_{\text {prim }}}\right) \cdot f_{a}
$$






Fig. 5. Simplified lumped parameter model of the experimental test bed.

with

- $f_{s}$ the measured force through the piezosensor

- $k_{\text {prim }}$ the primary stiffness: $1 / k_{\text {prim }}=1 / k_{c}+1 / k_{a}+1 / k_{s}$.

This equation shows that the stiffness $k_{p}$ of the secondary path has to be known in order to derive the transmitted force. However, in practice the representation of the complete secondary force transmission through the hinges, leaf springs and the secondary bearing as a stiffness is an oversimplification. Actually, the secondary path should be modeled as a general impedance instead of the spring in Fig. 5. Based on the measured frequency response functions between the piezoactuator force and the disturbance force on the one hand and the piezosensor signal and the plate acceleration on the other hand, a relatively simple impedance can be estimated, which represents the actual impedance quite accurately in the frequency range below $1 \mathrm{kHz}$. This impedance will be used in the control design in the next section.

To illustrate the beneficial effect of controlling the estimated transmitted force instead of the measured force, ideal control of the measured force (i.e. generating a piezoactuator force such that the measured force is equal to zero) is compared with ideal control of the estimated transmitted force (i.e. generating a piezoactuator force such that the estimated transmitted force is equal to zero). The achievable reductions of the plate vibrations can be calculated for both control strategies based on the measurement of some frequency response functions. The plate vibrations, which are strongly correlated to the radiated noise, are studied here because they are less affected by uncorrelated environmental noise and hence can be measured more accurately. The calculation procedure of the plate vibration reduction will be illustrated for the case of ideal control of the measured force. This calculation is based on the following three formulas:

$$
X_{1, \text { no control }}=H_{f_{d}-x_{1}} \cdot F_{d},
$$

which expresses the displacement of the plate $x_{1, \text { no control }}(t)$ as a function of the disturbance force when there is no control action;

$$
X_{s}=H_{f_{a}-x_{s}} \cdot F_{a}+H_{f_{d}-x_{s}} \cdot F_{d}=0,
$$

which states that the measured force is controlled to zero in the case of ideal control of the measured force; and

$$
X_{1, \text { meas force control }}=H_{f_{a}-x_{1}} \cdot F_{a}+H_{f_{d}-x_{1}} \cdot F_{d} \text {, }
$$

which expresses the displacement of the plate $x_{1 \text {,meas force control }}(t)$ as a function of the disturbance force in the case of ideal control of the measured force. $X_{1, \text { no control }}, X_{1 \text {,meas force control }}, F, F_{a}, X_{s}$ are the Fourier transforms of, respectively, $x_{1 \text {,no control }}(t)$, $x_{1 \text {, meas force control }}(t), f_{d}(t), f_{a}(t)$ and $x_{s}(t) . H_{f_{d}-x_{1}}, H_{f_{a}-x_{s}}, H_{f_{d}-x_{s}}$ and $H_{f_{a}-x_{1}}$ are the frequency response functions, respectively, between $f_{d}$ and $x_{1}$, between $f_{a}$ and $x_{s}$, between $f_{d}$ and $x_{s}$, and between $f_{a}$ and $x_{1}$. Combining Eqs. (2)-(4) leads to the following expression for the achievable reduction of the plate acceleration in the case of ideal control of the 
(a)
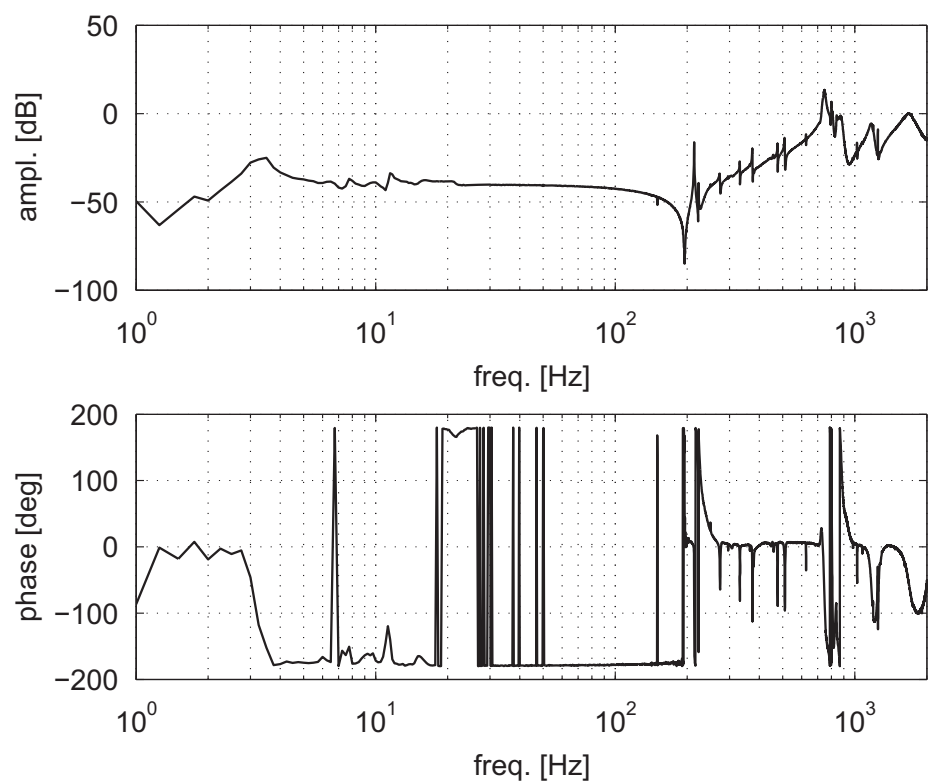

(b)

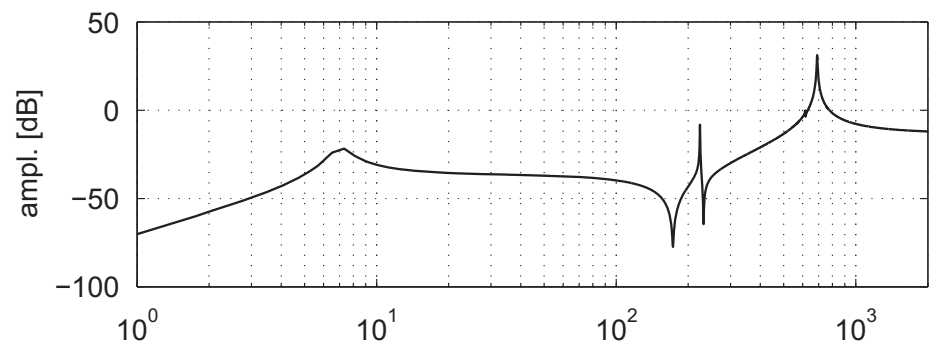

freq. $[\mathrm{Hz}]$

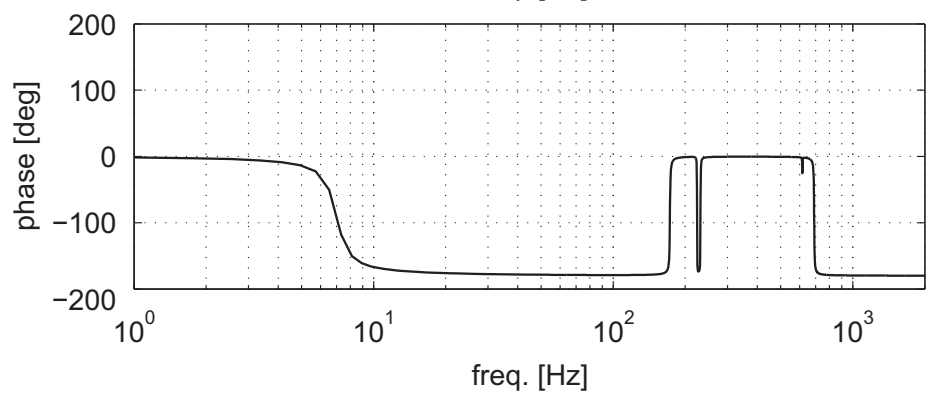

Fig. 6. Comparison of: (a) the measured driving point transfer function (between the shaker force on the shaft and the acceleration at the same point) and (b) the corresponding modeled transfer function.

measured force:

$$
\frac{X_{1, \text { meas force control }}}{X_{1, \text { no control }}}=1-\left(\frac{H_{f_{a}-x_{1}}}{H_{f_{a}-x_{s}}}\right)\left(\frac{H_{f_{d}-x_{1}}}{H_{f_{d}-x_{s}}}\right)^{-1} .
$$

A similar expression can be derived in the case of ideal control of the estimated transmitted force:

$$
\frac{X_{1, \text { est transm force control }}}{X_{1, \text { no control }}}=1-\left(\frac{H_{f_{a}-x_{1}}}{H_{f_{a}-f_{\text {trans }}}}\right)\left(\frac{H_{f_{d}-x_{1}}}{H_{f_{d}-f_{\text {trans }}}}\right)^{-1} .
$$

For both ideal control strategies, the achievable reductions of the plate acceleration (calculated by Eqs. (5) and (6)) are plotted in Fig. 7. It is clear that both strategies lead to a significant vibration reduction around $700 \mathrm{~Hz}$, which actually corresponds to the resonance frequency of the shaft on the bearing stiffnesses. At this resonance, force is transmitted 


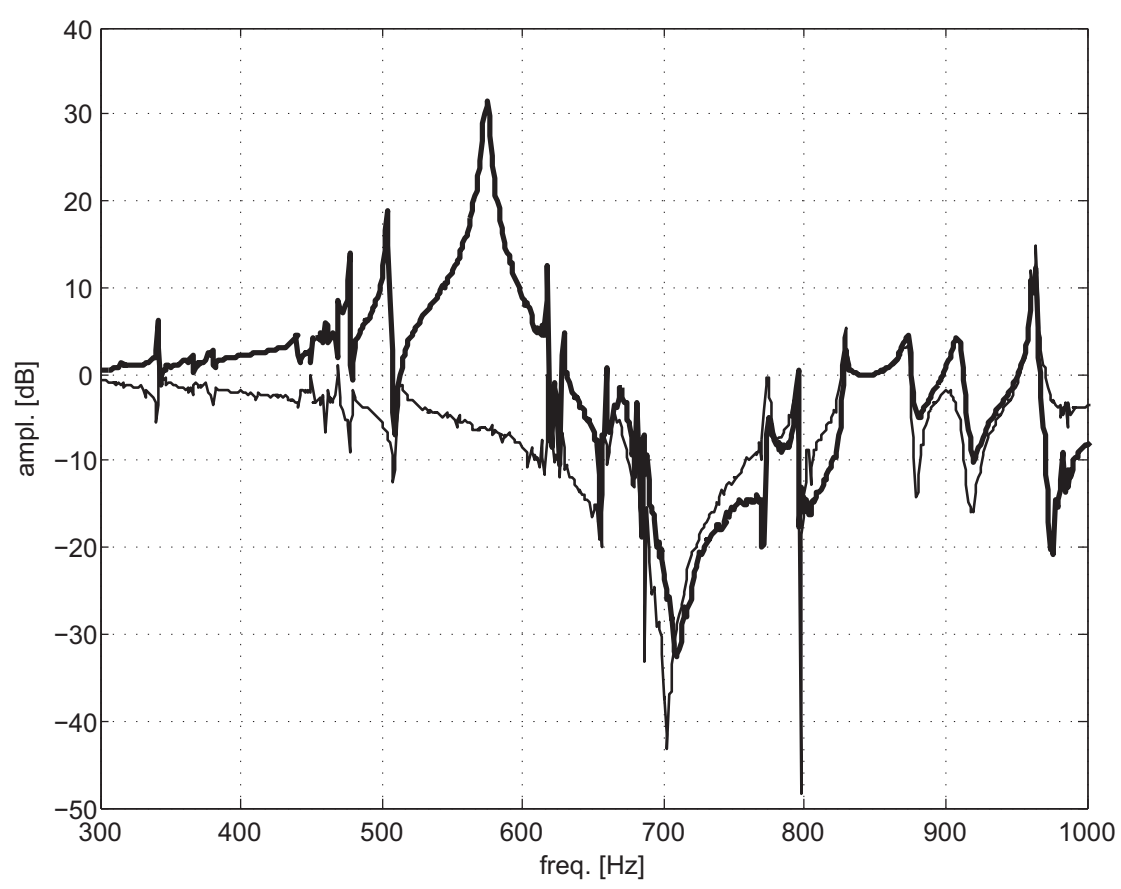

Fig. 7. Comparison of the achievable reduction of the plate vibrations in the case of a cancelation of the measured force (thick line) and the estimated transmitted force (thin line).

efficiently from the shaft to the frame in the passive test bed such that a great improvement can be realized by the introduction of the active system. Moreover, Fig. 7 also shows that control of the transmitted force generally leads to a better reduction of the plate acceleration than control of the measured force. This is especially true around $570 \mathrm{~Hz}$, which corresponds to the shaft resonance when the primary transmission path is canceled by ideal control of the measured force. Therefore, in the next section a first controller will be developed which uses the estimated transmitted force as the error signal instead of the measured force. Below $500 \mathrm{~Hz}$, the control of the transmitted force leads only to small reductions of the plate acceleration. At these lower frequencies, the generated piezoactuator force is mainly used to compensate the force created by the extension of the secondary spring $k_{p}$. Consequently, the generated transmitted force is only a small part of the generated piezoactuator force, which is equal to the measured force by the piezosensor. This makes it hard to estimate the transmitted force accurately based on the measured force, which explains the poor performance of the transmission force controller at low frequencies. Therefore in the fifth section a second controller, which uses the frame acceleration as the error signal and focusses on the frequency range below $500 \mathrm{~Hz}$, will be added to the transmitted force controller in order to achieve noise reduction over the complete frequency range below $1 \mathrm{kHz}$.

\section{Force control}

In this section the performance of three controllers, which aim at canceling the estimated transmitted force, is compared. Firstly, a simple robust feedback controller is designed. In a second step, the performance of this controller is further enhanced by the addition of an adaptive repetitive controller (RC [29,30]). An RC controller can significantly suppress the disturbance frequency content at a fundamental frequency and its harmonics, and is therefore very suitable to control the periodic gear noise. Although repetitive control is not often associated with acoustic control, similar performance can be achieved by adaptive repetitive control and filtered-X LMS control for the suppression of periodic disturbances [19]. Furthermore, the stability criterion is similar in both algorithms. In this paper, the repetitive control strategy was chosen since this strategy leaves more freedom in the design of the control filters.

\subsection{Feedback control}

To simplify the initial control design, only the piezoactuator in the horizontal direction is used in the first experiments. To test the performance of the developed controllers in this case, a horizontal disturbance force is executed on the shaft. For the design of the feedback controller in this direction, the loopshaping method was used. Based on the measured frequency response function between the voltage to the piezoactuator amplifier and the estimated transmitted force in the 
horizontal direction (Fig. 8), it was decided to introduce 1 pole in the controller at $1 \mathrm{kHz}$ for stability reasons. The controller gain is set such that a gain margin of $6 \mathrm{~dB}$ is obtained to guarantee sufficient robustness. Fig. 9 shows the sensitivity of this SISO controller: a reduction of the measured force signal in the horizontal direction can theoretically be obtained between 300 and $1500 \mathrm{~Hz}$.
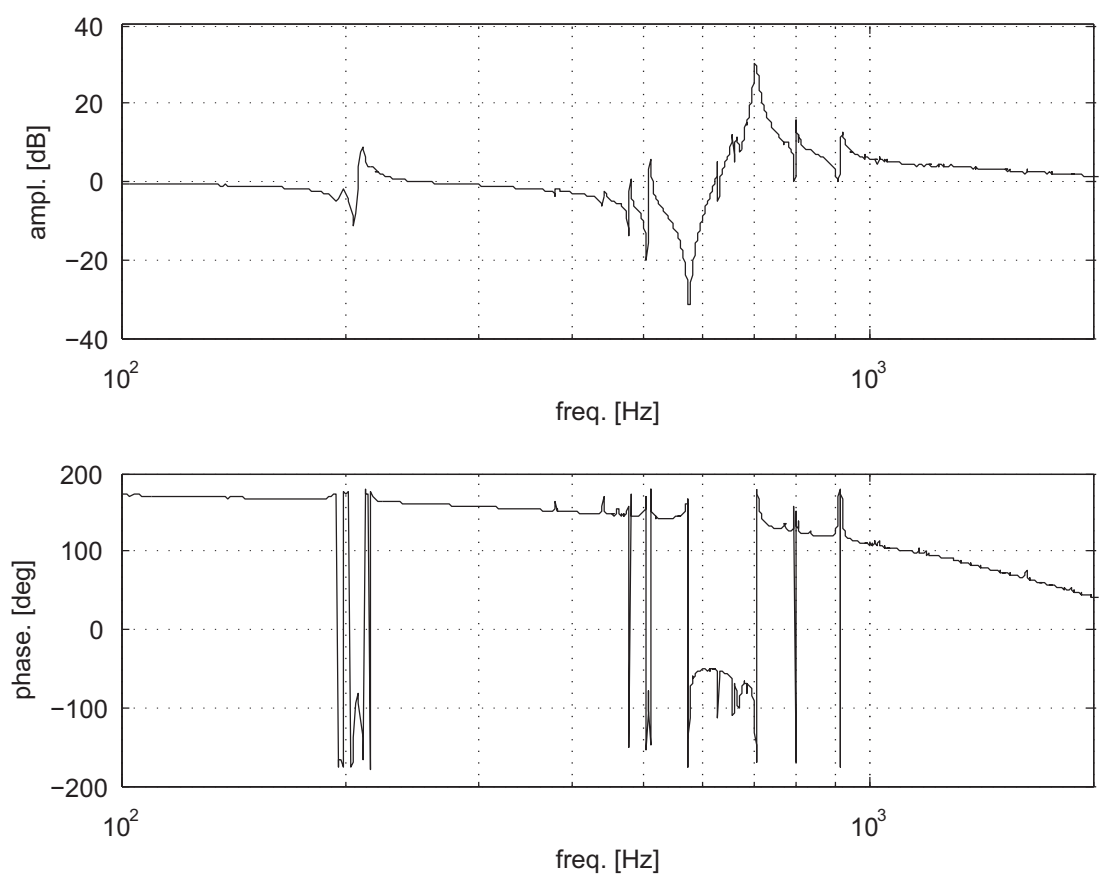

Fig. 8. The frequency response function of the first controlled system between the voltage to the piezoactuator and the estimated transmitted force.

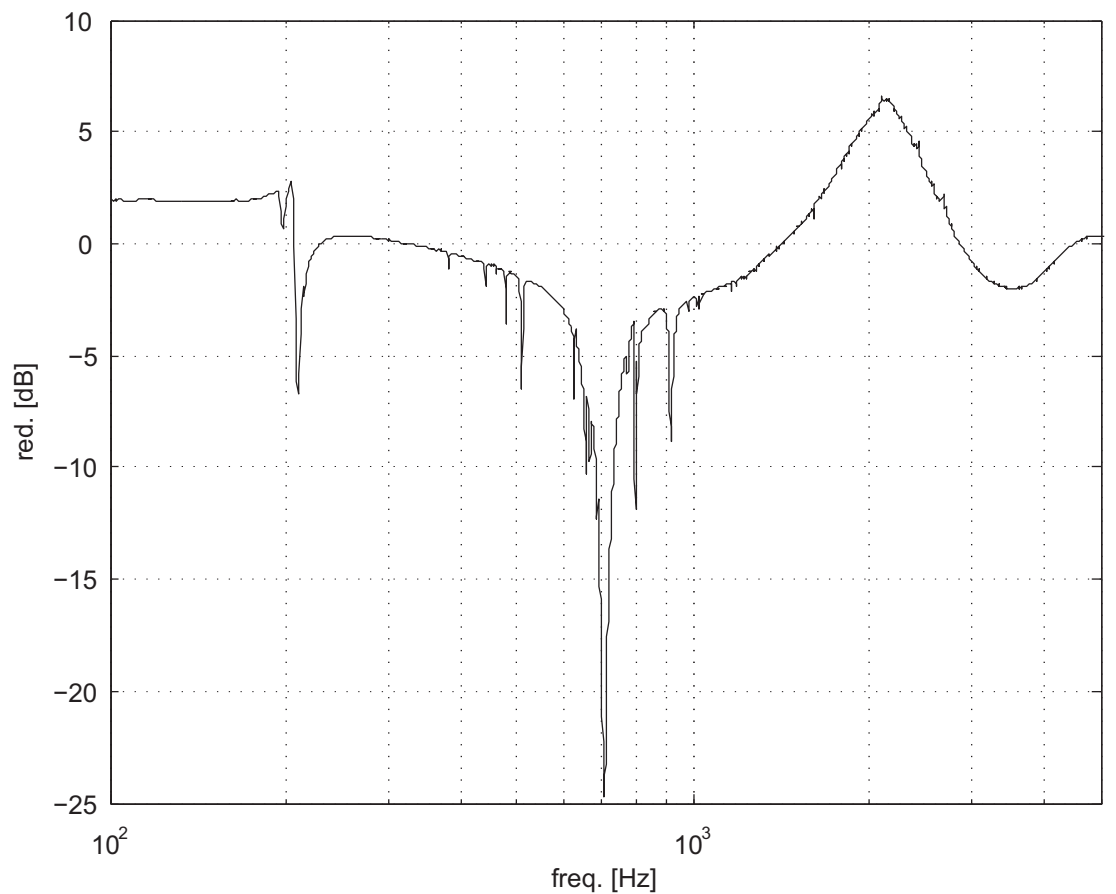

Fig. 9. The theoretically achievable reduction of the measured force signal (horizontal direction) by the designed feedback controller ( $\left.F_{\text {measured, with control }} / F_{\text {measured, without control }}\right)$. 
(a)

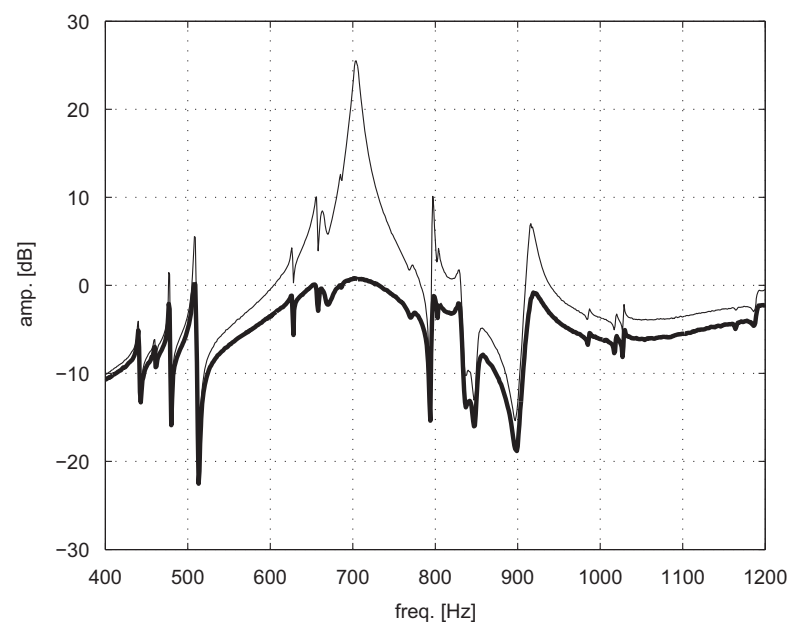

(b)

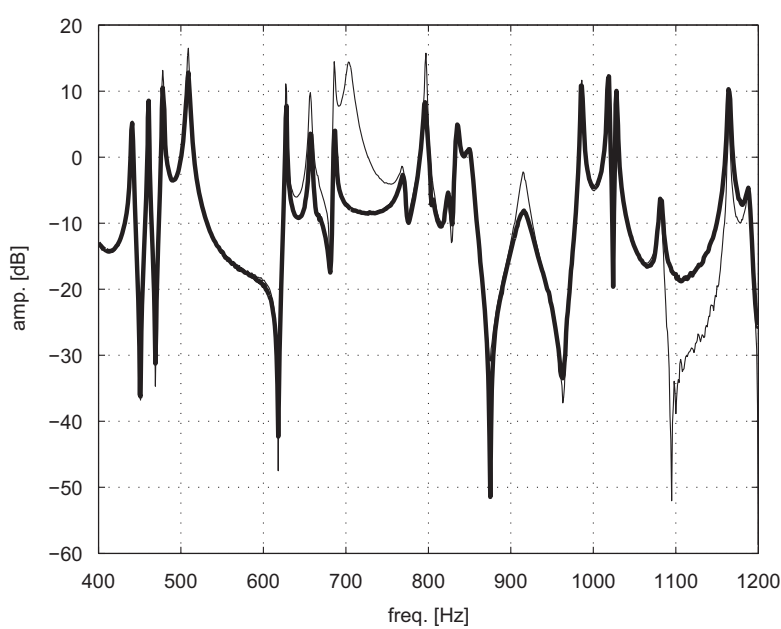

(c)

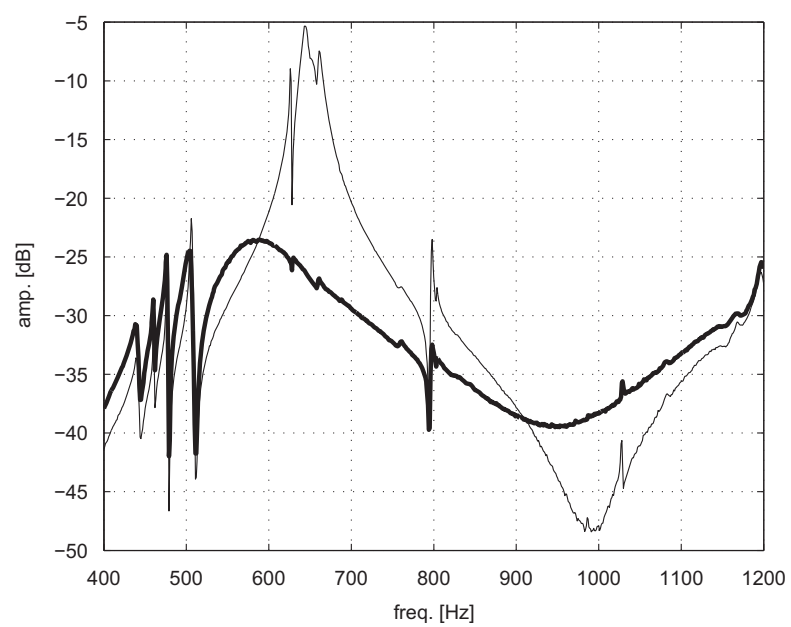

Fig. 10. The autopower spectra of (a) the estimated transmitted force, (b) the plate acceleration and (c) the shaft vibrations without control (thin line) and with force feedback control (thick line).

The designed controller is practically tested in an experiment, where the shaker exerts a broadband disturbance force on the shaft. In Fig. 10(a) the autopower spectra of the estimated transmitted force are compared when the feedback controller is turned on and off, while in Fig. 10(b) the autopower spectra of the plate acceleration are compared. Around $700 \mathrm{~Hz}$, i.e. the shaft resonance frequency, a high reduction is obtained in the estimated transmitted force as well as in the plate vibration (which is strongly correlated to the radiated noise). This confirms the observation in the previous section that above $500 \mathrm{~Hz}$ a reduction of the transmitted force leads to a reduction of the plate vibrations. Furthermore, Fig. 10(c) shows that also the shaft vibration is significantly reduced around its resonance frequency. This shows that actually due to the introduction of the active control system damping is added to the structure, which is also beneficial with respect to fatigue and failure of the machine.

A SISO controller, similar to the one in the horizontal direction, is also developed to control the piezoactuator in the vertical direction. This controller uses the estimated transmitted force in the vertical direction to calculate the control signal in this direction. Similar results could be obtained as in the horizontal direction. Experiments have also been carried out with a simultaneous control action in the horizontal and vertical direction to check the coupling effects of the two controllers. Due to the attention in the mechanical design to achieve a strong decoupling of the horizontal and vertical active system, no stability problems were encountered and a good performance could be achieved in both directions.

\subsection{Repetitive control}

In industrial rotating machinery applications, the disturbance is often periodic (e.g. due to gear meshing) with a major frequency content at the harmonics of the rotational speed. In an adaptive repetitive control algorithm, an estimation of 
the disturbance period is used to improve the control performance at these harmonics of the rotational speed. In contrast with the filtered-X LMS algorithm, which can be applied for the control of periodic as well as stochastic disturbances, RC can only be used to suppress periodic disturbances. Fig. 11 shows the conventional (non-adaptive) discrete time RC scheme, where $P_{\text {prim }}$ is the primary plant, relating the disturbance force $f_{d}$ to the output $y$ and $P_{\text {sec }}$ is the secondary plant, relating the piezoactuator input voltage $u$ to output $y$. The output $y$ is the measured error signal, in this case the estimated transmitted force $f_{\text {trans. }}$. Essential to the repetitive controller is the presence of a memory loop with delay operator $z^{-p}$, with $p$ the number of samples in one period of the disturbance. Due to this memory loop, a disturbance rejection can be obtained at the fundamental frequency $\omega_{p}\left(=2 \pi /\left(p \cdot T_{s}\right)\right.$ with $T_{s}$ the sample time $)$ and its harmonics. In a conventional RC controller, the disturbance period is a constant, which should be known beforehand and be equal to an integer number ( $p$ ) of samples. In the application of rotating machinery, however, often various rotational speeds occur and therefore the repetitive controller should be adaptive, with its fundamental frequency following the rotational speed. For the adaptation of the algorithm, a trigger signal must be available such that the parameter $p$, which determines the fundamental frequency, can be adjusted (Fig. 12). In industrial situations, a tacho signal, which measures the rotational motor speed, can be used as trigger signal. Since the disturbance force in the test bed is generated by a shaker, in this case the fundamental disturbance frequency can easily be derived from the control signal to this shaker.

The learning filters $Q(z)$ and $L(z)$ (Fig. 12) should be designed such that stability of the RC algorithm is guaranteed and a good performance is obtained in the frequency range of interest. Since, as mentioned in Section 3, a cancelation of the estimated transmitted force through the piezoactive bearing will lead to noise reduction only above $500 \mathrm{~Hz}$, the frequency band between 500 and $1000 \mathrm{~Hz}$ is selected as the target frequency band for the RC algorithm. A strategy, which is adapted to structural applications with a high modal density and lowly damped resonances, is applied for the design of suitable learning filters [31]. This paper will not elaborate on the design procedure but refers to [31] for further details.

The performance of the developed adaptive repetitive controller is demonstrated in Fig. 13 in the case of a sinusoidal disturbance force excitation by the shaker at $700 \mathrm{~Hz}$. In Fig. 13(a), the transmitted force is shown when the RC controller is activated: after the convergence of the algorithm, which takes approximately $2 \mathrm{~s}$, a reduction of $33 \mathrm{~dB}$ of the transmitted force is obtained by the RC controller. In Fig. 13(b), it is shown that this reduction of the transmitted force also leads to a reduction of the plate vibrations: a vibration reduction of $25 \mathrm{~dB}$ is achieved after convergence. During the convergence process of the RC controller, an undesired amplification of the transmitted force and the plate vibrations shows up. A repetitive controller negatively affects the closed-loop performance for non-periodic inputs, such as this transient response when the controller is turned on. Due to the Bode sensitivity integral [32], pushing the sensitivity of RC controller down to zero at the multiples of the fundamental disturbing frequency is paid for by an increased sensitivity at intermediate frequencies. This is illustrated in Fig. 14, which plots the sensitivity of the developed RC controller for a fundamental frequency of $50 \mathrm{~Hz}$. Due to the low sensitivity at the harmonics of $50 \mathrm{~Hz}$, a strong error reduction can be obtained by the RC action at these frequencies as expected. However, at the intermediate frequencies, the sensitivity becomes bigger than 1 , resulting in an error amplification of even more than $10 \mathrm{~dB}$ at some frequencies. During the transient behavior when the RC

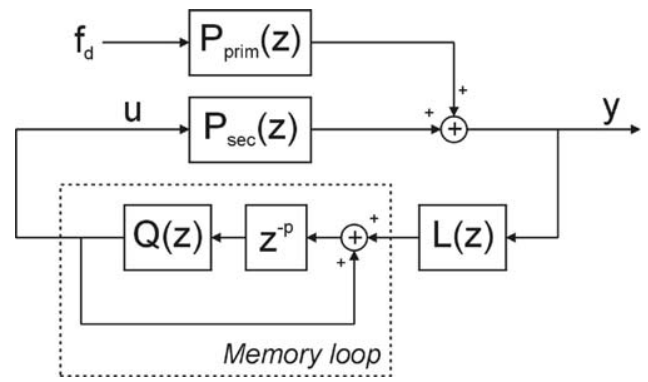

Fig. 11. The conventional block scheme of repetitive control (RC).

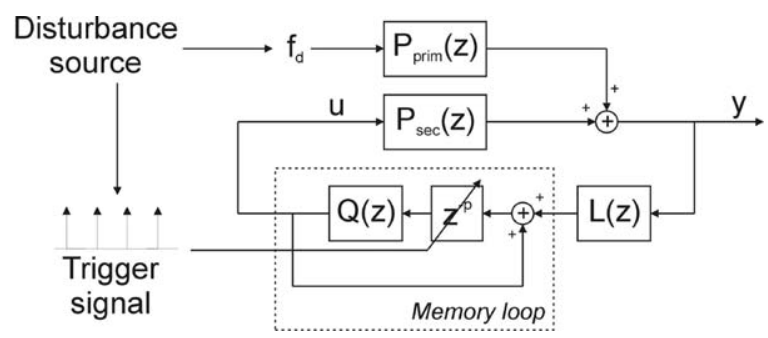

Fig. 12. The block scheme of adaptive repetitive control. 
(a)

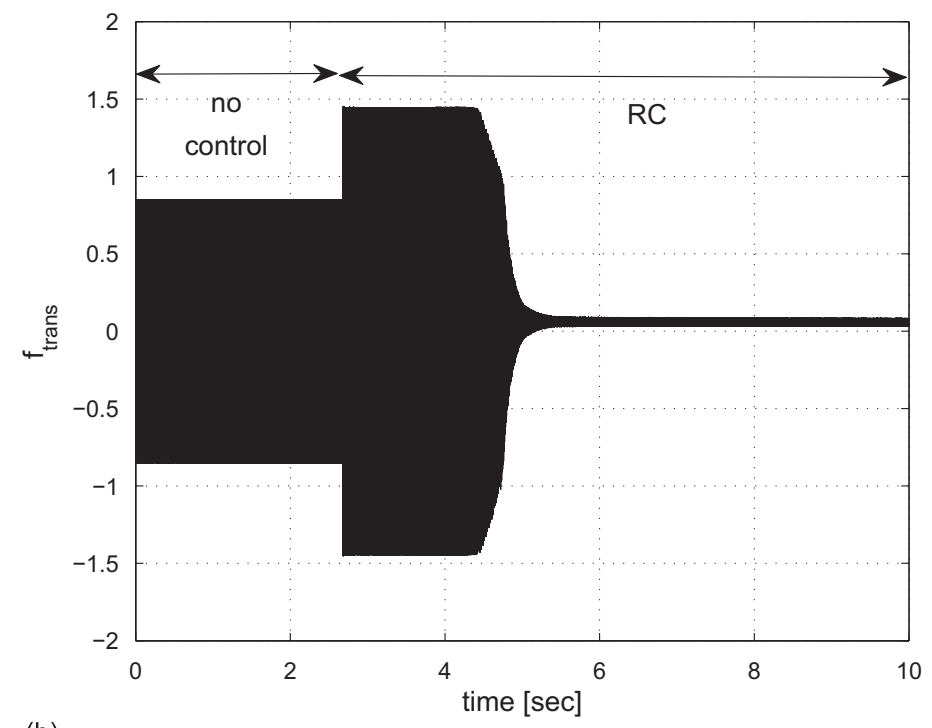

(b)

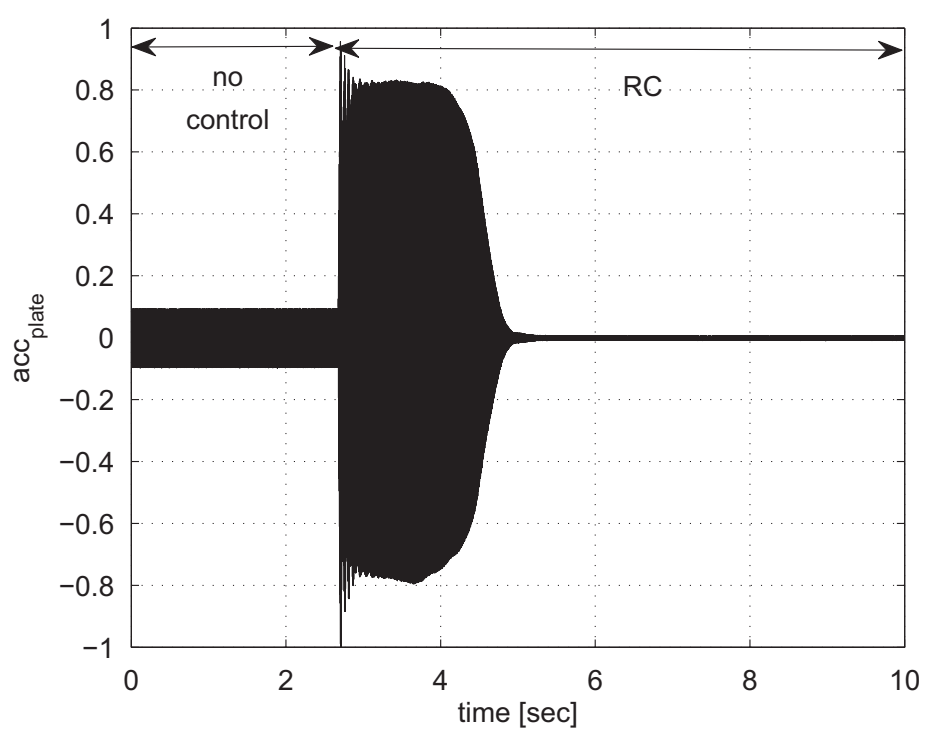

Fig. 13. The effect of the RC action on (a) the estimated transmitted force and (b) the plate vibrations.

controller is turned on, a broad frequency band is excited. The high sensitivity at certain frequencies leads to the undesired transient amplification of the transmitted force. To deal with this disadvantage, higher-order RC [33] can be used, where not only the error signal of the last period but also of previous periods is processed to calculate the control signal of the actual period. By optimizing the learning filters in the higher-order RC design, an optimal trade-off can be made between the behavior during convergence and the error reduction after convergence of the RC controller.

\subsection{Hybrid control}

The control strategies discussed in the two previous paragraphs, feedback and RC, both have certain benefits. The feedback controller can not only cancel repetitive but also random disturbances and is immediately effective without a time interval for convergence. On the other hand, the RC controller can seriously enhance the control performance at the harmonics of the fundamental disturbing frequency in comparison with the feedback controller, but only achieves its final performance after a certain convergence time. Therefore, a combined system with a feedback and an RC algorithm (Fig. 15) is applied for the control of the piezoactive bearing. In this control scheme, an RC algorithm is developed for the active 


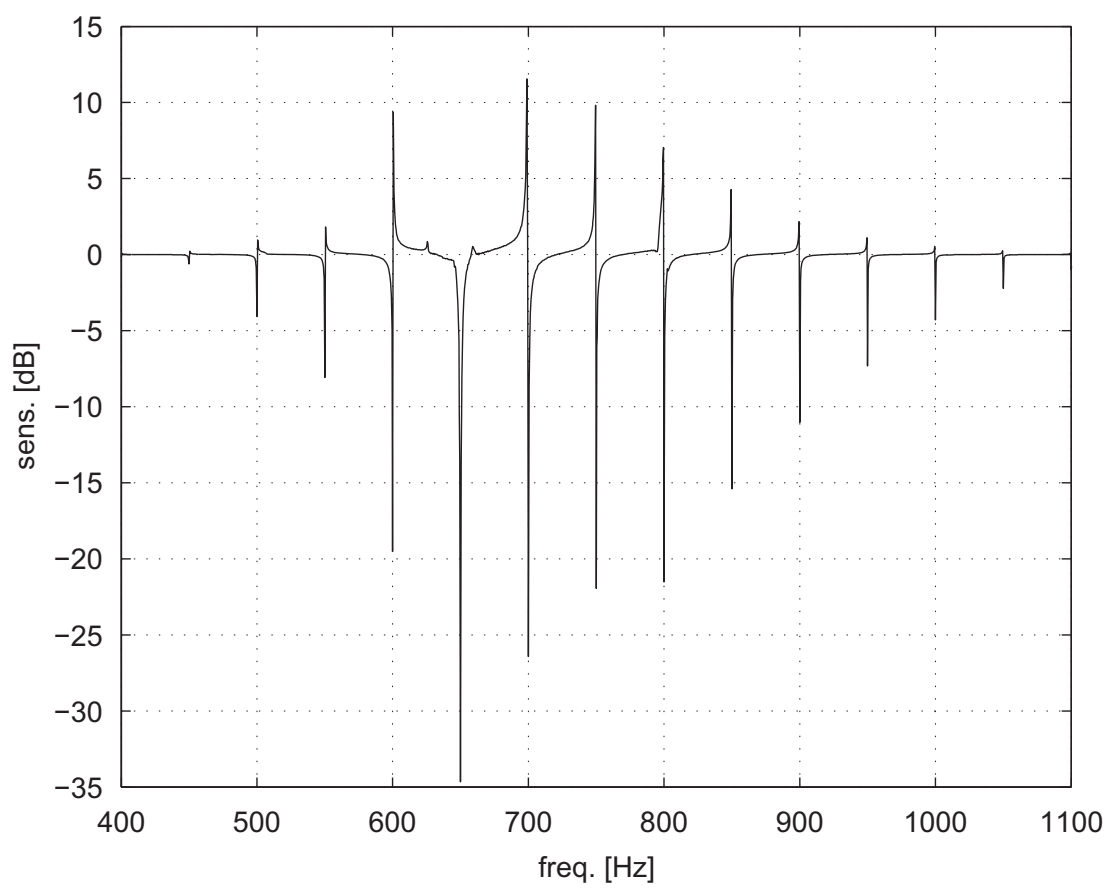

Fig. 14. The sensitivity of the RC force controller (for a fixed fundamental frequency of $50 \mathrm{~Hz}$ ).

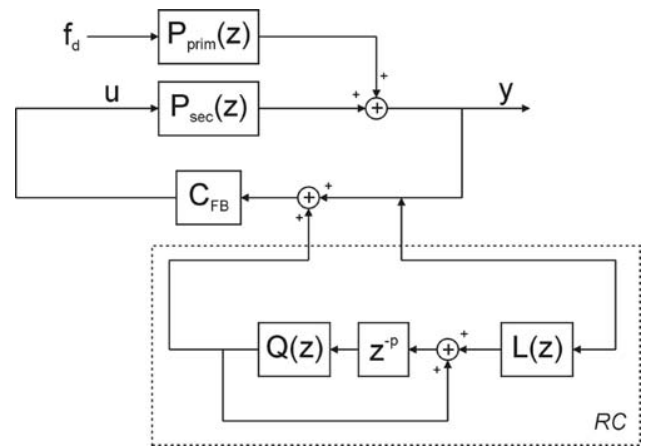

Fig. 15. The hybrid control scheme combining a feedback controller and an RC controller.

system, which is already controlled by the feedback algorithm from Section 4.1. The feedback controller creates a broadband force reduction, while the RC controller is only used to further suppress the remaining force at the harmonics of a certain fundamental frequency.

Fig. 16(a), which compares the reduction of the transmitted force in the case of repetitive disturbances obtained by the feedback controller with the reduction obtained by the hybrid controller, shows that the introduction of the RC controller significantly increases the performance of the control system. In Fig. 16(b), the corresponding noise reduction of the hybrid controller is compared with the reduction of the feedback controller. It is clear that, due to the introduction of the RC controller, also an additional noise reduction (up to $10 \mathrm{~dB}$ ) can be achieved.

\section{Frame acceleration control}

As mentioned in Section 3, a cancelation of the estimated transmitted force will only lead to a significant reduction of the radiated noise level above $500 \mathrm{~Hz}$. Consequently, to apply the piezoactive bearing also at lower frequencies, a second error sensor and a corresponding controller are required. An accelerometer on the outer part of the active bearing that is fixed to the frame, is chosen as the most appropriate additional error sensor. An error sensor on the active bearing implies that the modularity of the complete active unit is maintained, which improves its applicability. Furthermore, from the 
(a)

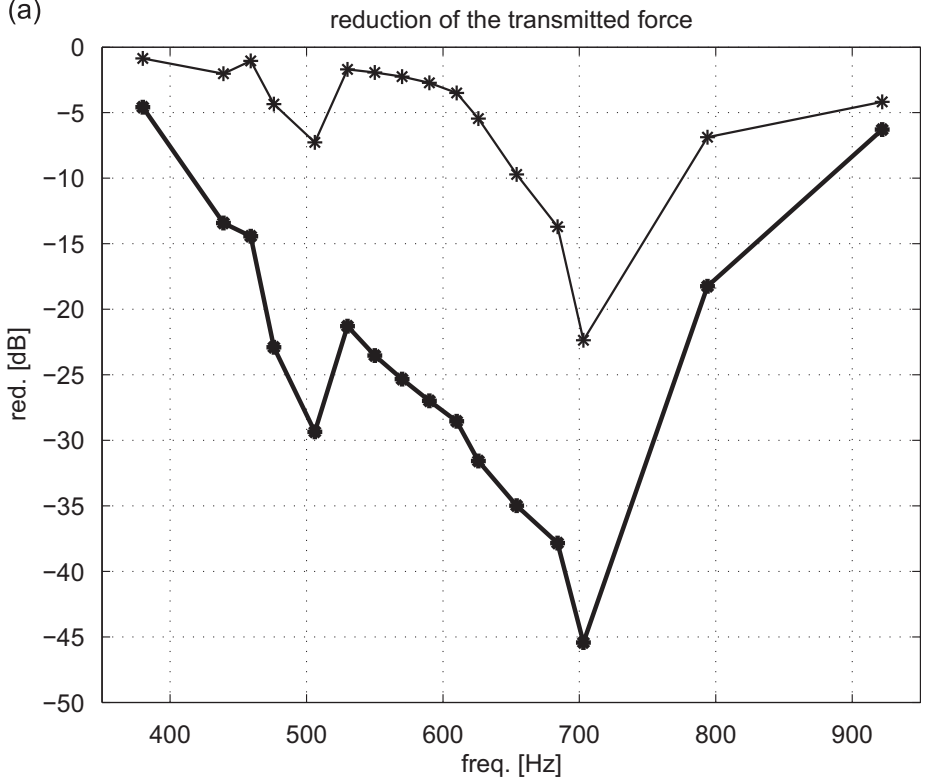

(b)

noise reduction

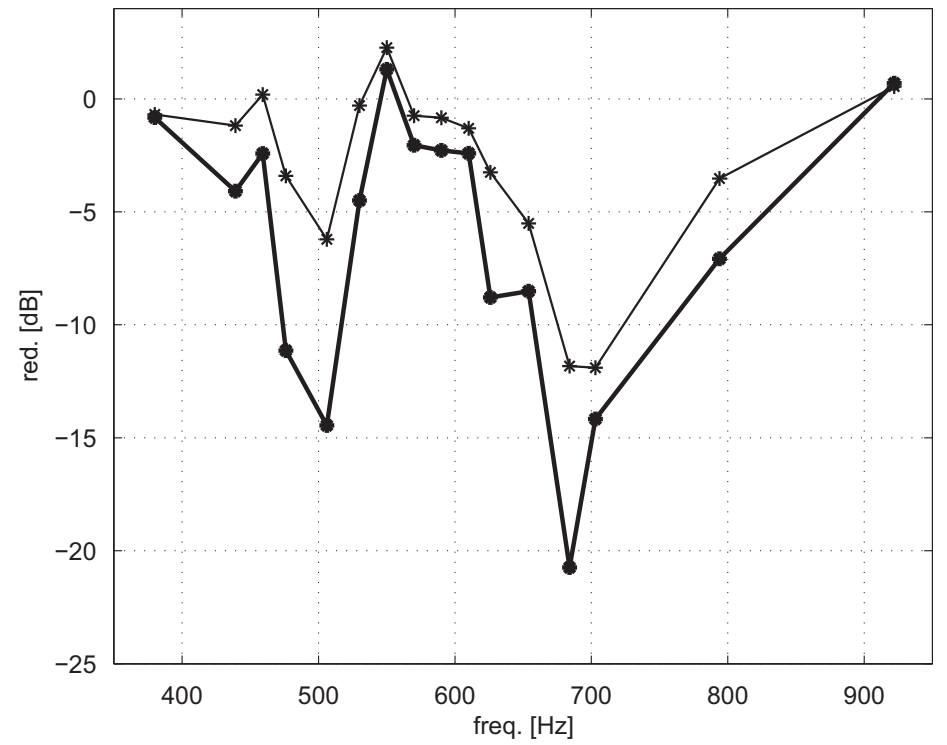

Fig. 16. The reduction of (a) the estimated transmitted force and (b) the radiated noise obtained, respectively, by the feedback controller (thin line) and the RC controller (thick line).

model in Fig. 5, it can be derived that frame acceleration control is equivalent with plate vibration control and noise control from a theoretical point of view: a cancelation of the frame vibrations also leads to a cancelation of the plate vibrations and the radiated noise. To validate the correctness of this theoretical statement in practice, the reduction of the plate vibrations is calculated in the case of a perfect cancelation of the frame acceleration. This is done in a similar way as in Eqs. (5) and (6) based on a measurement of different frequency response functions (between the shaker force and the piezoactuator force on the one hand and the frame acceleration and the plate acceleration on the other hand). Fig. 17 shows that a cancelation of the frame acceleration actually leads to a reduction of the plate acceleration, at least below $800 \mathrm{~Hz}$. Above $800 \mathrm{~Hz}$, the frame modes are excited in a different way by the shaker and the piezoactuator, such that a local cancelation of the acceleration at one point on the frame will not necessarily lead to a global reduction of the whole frame acceleration.

In the case of frame acceleration control, the dynamics of the controlled system between the piezoactuator(s) and the accelerometer on the frame are very complex: Fig. 18 shows the frequency response functions of the controlled system between the piezoactuator control force and the acceleration on the frame. Due to the high amount of resonance 


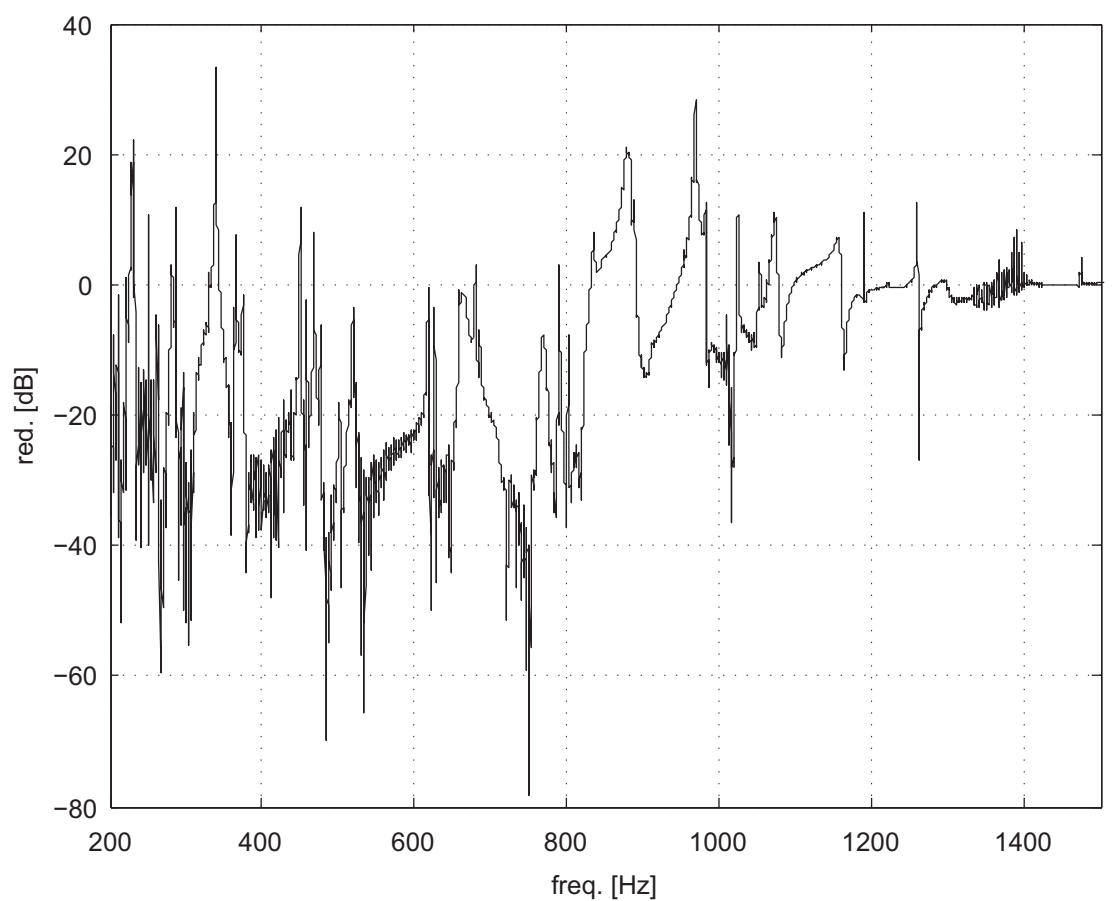

Fig. 17. The theoretically achievable reduction of the plate vibrations, when the frame acceleration is completely canceled at the controlled location.

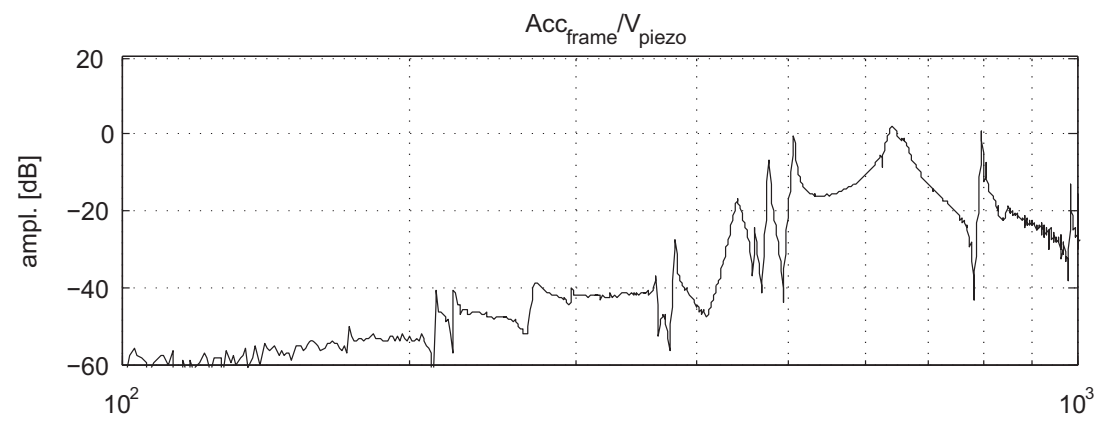

freq. $[\mathrm{Hz}]$

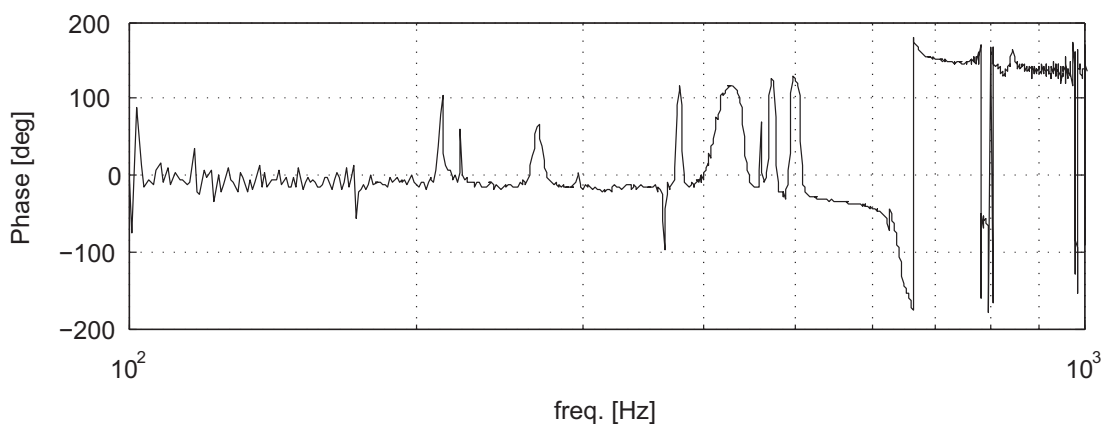

Fig. 18. The frequency response function of the second controlled system between the voltage to the piezoactuator and the frame acceleration.

frequencies in the controlled frequency range, it is very difficult to design a stable and efficient feedback controller for this system. Even if a suitable higher-order controller could be found, the resulting control system would not be sufficiently robust and would require too much computational power for a practical implementation. Therefore, instead of a hybrid control system, only the potential of an RC controller, which can deal with more complicated system dynamics, can be 


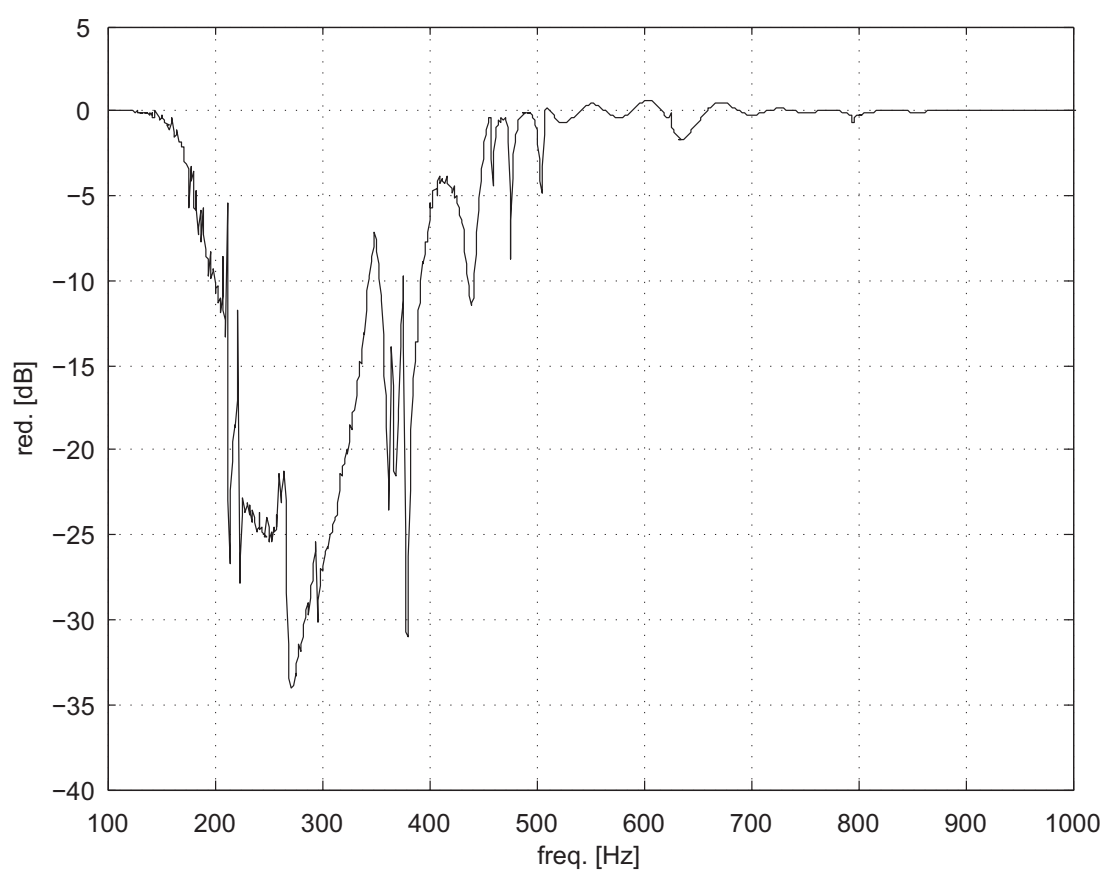

Fig. 19. The theoretically achievable reduction of the frame acceleration by the developed RC controller.

tested in this case and, consequently, only a tonal (and no broadband) vibration reduction can be realized. Since the noise, radiated by the plate above $500 \mathrm{~Hz}$ could already be reduced by force control as explained in the previous section, the focus in the design of this repetitive controller $(\mathrm{RC})$ for the frame acceleration is on the frequency range below $500 \mathrm{~Hz}$. As lower frequency limit of the controller, $200 \mathrm{~Hz}$ was chosen, since significant noise radiation occurs only starting from around the lowest eigenmode of the plate $(210 \mathrm{~Hz})$. For the control design, the same strategy from [31] is applied, which was also used in the case of the force control algorithm.

The theoretically achievable reduction of the frame acceleration by the developed RC algorithm at the harmonics of the base frequency can be calculated based on the frequency response functions of the controlled system (between the piezoactuator force and the frame acceleration) and the learning filters $Q(z)$ and $L(z)$. The result is shown in Fig. 19: a theoretical reduction of more than $10 \mathrm{~dB}$ can be created by the designed repetitive controller over almost the whole frequency band between 200 and $500 \mathrm{~Hz}$. To illustrate the potential of this repetitive controller, an experiment is carried out with a sinusoidal disturbance force excitation at a plate resonance frequency ( $380 \mathrm{~Hz}$ ). The frame and plate acceleration are shown in the time domain in Figs. 20(a)-(c), when the RC controller is turned on. Due to the high noise level, which is picked up by the error accelerometer on the frame, the actually achieved reduction of the frame acceleration of $9 \mathrm{~dB}$ at $380 \mathrm{~Hz}$ cannot be observed in Fig. 20(a), which plots the frame acceleration. To show this reduction of the frame acceleration at $380 \mathrm{~Hz}$, the frame acceleration is filtered by a bandpass filter, which passes the frequency range between 370 and $390 \mathrm{~Hz}$ (Fig. 20(b)). The effect of the repetitive controller is also clearly visible in the plate acceleration (Fig. 20(c)). After a convergence process, which lasts approximately $3 \mathrm{~s}$, the disturbing frequency is suppressed by $7 \mathrm{~dB}$. This shows that a local cancelation of the frame acceleration leads also to a reduction of the plate acceleration, and thus of the radiated noise.

\section{Summary and conclusion}

In order to reduce the structure borne noise radiation of rotating machinery, a modular active bearing has been designed to isolate the radiating structure housing from the disturbance that stems from the rotating device. Because all actuators and sensors are integrated, the active bearing is a modular unit, which improves its applicability. This active bearing has been tested on an experimental test bed leading to a significant reduction of noise and vibrations below $1 \mathrm{kHz}$. Above $500 \mathrm{~Hz}$, hybrid (feedback + repetitive) control of the transmitted force through the bearing is applied. Below $500 \mathrm{~Hz}$, repetitive control of the frame acceleration is implemented. At the most important resonance frequencies of the system, a noise reduction of more than $10 \mathrm{~dB}$ is achieved. This demonstrates the technical feasibility of piezo-based active structural acoustic control for rotating machinery. However, in practical applications, the secondary force transmission through the passive bearing, which is limited in the studied test set-up due to the placement of the disturbing shaker force, will be 
(a)



(b)

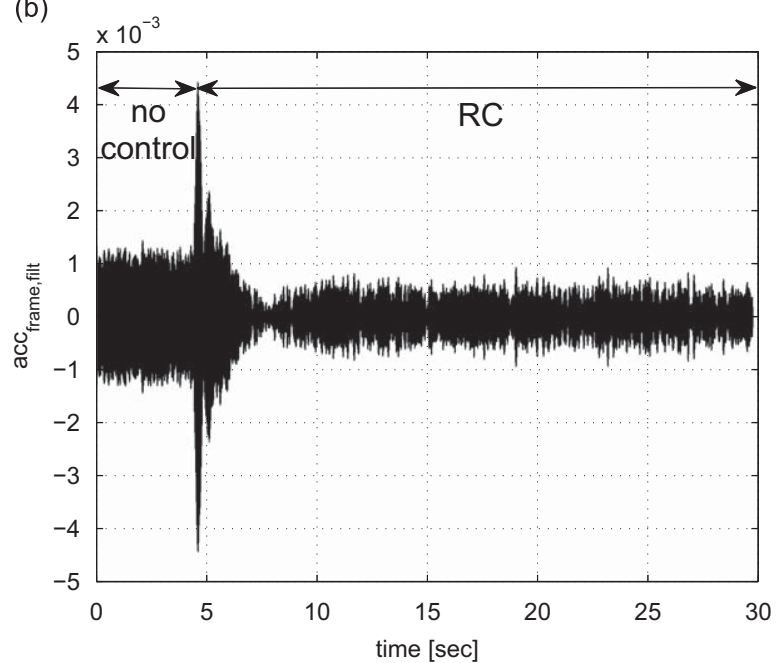

(c)



Fig. 20. The effect of the RC action on (a) the frame acceleration, (b) the filtered frame acceleration and (c) the plate acceleration.

more pronounced. Therefore, the effect of this secondary force transmission path on the performance of the active bearing should be investigated in future work. A possible solution is the introduction of a second active bearing, which replaces the passive bearing.

\section{Acknowledgment}

The research of B. Stallaert is financed by a scholarship of the Institute for the Promotion of Innovation through Science and Technology in Flanders (IWT Vlaanderen).

\section{References}

[1] C.R. Fuller, C.H. Hansen, S.D. Snyder, Active control of sound radiation from a vibrating rectangular panel by sound sources and vibration inputs: an experimental comparison, Journal of Sound and Vibration 145 (1991) 195-215.

[2] W. Dehandschutter, The reduction of structure-borne noise by active control of vibration, Ph.D. Thesis, KU Leuven, Department of Mechanical Engineering, Division of Production Engineering, Machine Design and Automation, 1997.

[3] P. Gardonio, S.J. Elliott, Smart panels for active structural acoustic control, Smart Materials and Structures 13 (6) (2004) 1314-1336.

[4] K. Henrioulle, W. Dehandschutter, P. Sas, Design of an active noise control system using a distributed actuator, Flow, Turbulence and Combustion 61 (1999) 189-209.

[5] S.J. Elliott, Active control of structure borne noise, Journal of Sound and Vibration 177 (1994) 651-673.

[6] J.D. Smith, Gear Noise and Vibration, Marcel Dekker, New York, 1999. 
[7] Y.H. Guan, M. Li, T.C. Lim, W.S. Shepard Jr., Comparative analysis of actuator concepts for active gear pair vibration control, Journal of Sound and Vibration $269(1-2)(2004)$ 273-294.

[8] B. Rebbechi, C. Howard, C. Hansen, Active control of gearbox vibration, in: Proceedings of Active, vol. 99, 1999, pp. $295-303$.

[9] Y.H. Guan, T.C. Lim, W.S. Shepard Jr., Experimental study on active vibration control of a gearbox system, Journal of Sound and Vibration 282 (3-5) (2005) 713-733.

[10] M. Li, T.C. Lim, W.S. Shepard Jr., Y.H. Guan, Experimental active vibration control of gear mesh harmonics in a power recirculation gearbox system using a piezoelectric stack actuator, Smart Materials and Structures 14 (5) (2005) 917-927.

[11] A.B. Palazzolo, S. Jagannathan, A.F. Kascak, G.T. Montague, L.J. Kiraly, Hybrid active vibration control of rotorbearing systems using piezoelectric actuators, Journal of Vibration and Acoustics 115 (1993) 111-119.

[12] G. Schweitzer, Active magnetic bearings - chances and limitations, in: 6th International Conference on Rotor Dynamics, 2002.

[13] K. Tammi, Active control of radial rotor vibrations-identification, feedback, feedforward, and repetitive control methods, Ph.D. Thesis, VTT, Department of Automation and Systems Technology, 2007.

[14] Y.-Y. He, S. Oi, F.-L. Chu, H.-X. Li, Vibration control of a rotor-bearing system using shape memory alloy: I. Theory, Smart Materials and Structures 16 (2007) 114-121.

[15] Y.-Y. He, S. Oi, F.-L. Chu, H.-X. Li, Vibration control of a rotor-bearing system using shape memory alloy: II. Experimental study, Smart Materials and Structures 16 (2007) 122-127.

[16] A. Alizadeh, C. Ehmann, U. Schönhoff, R. Nordmann, Robust active vibration control of flexible rotors using piezo actuators as active bearing, in: International Symposium on Stability Control of Rotating Machinery, Gdansk, August, 2003.

[17] C.H. Hansen, Understanding Active Noise Cancellation, Spon Press, London, UK, 2001.

[18] S.J. Elliott, Signal Processing for Active Control, Academic Press, London, UK, 2001.

[19] B. Stallaert, G. Pinte, S. Devos, W. Symens, J. Swevers, P. Sas, Filtered-X LMS vs repetitive control for active structural acoustic control of periodic disturbances, in: International Conference on Noise and Vibration Engineering Leuven, Leuven, Belgium, 2008.

[20] A. Preumont, Vibration Control of Active Structures, An Introduction, Kluwer Academic Publishers, Dordrecht, 2002

[21] R.L. Clark, K.D. Frampton, D.G. Cole, Phase compensation for feedback control of enclosed sound fields, Journal of Sound and Vibration 195 (1996) $701-718$.

[22] L. Meirovitch, Dynamics and Control of Structures, Wiley, New York, USA, 1990.

[23] B. Bingham, M.J. Atalla, N.W. Hagood, Comparison of structural-acoustic control designs on an active composite panel, Journal of Sound and Vibration 244 (5) (2001) 761-778.

[24] P.R. Fraanje, M. Verhaegen, N.J. Doelman, A.P. Berkhoff, MIMO H-2 optimal feedback controller estimation for a vibrating plate using subspace model identification, in: International Conference on Noise and Vibration Engineering Leuven, Leuven, Belgium, 2002.

[25] J.H. Kim, S.B. Choi, C.C. Cheong, S.S. Han, J.K. Lee, H-infinity control of structure-borne noise of a plate featuring piezoceramic actuators, Journal of Sound and Vibration 8 (1) (1999) 1-12.

[26] D. Childs, Turbomachinery Rotordynamics: Phenomena Modeling and Analysis, Wiley, New York, USA, 1993.

[27] J.M. Vance, Rotordynamics of Turbomachinery, Wiley, New York, USA, 1987.

[28] S. Devos, Development of fast, stiff and high-resolution piezoelectric motors with integrated bearing driving functionality, Ph.D. Thesis, KU Leuven, Department of Mechanical Engineering, Division of Production Engineering, Machine Design and Automation, 2006.

[29] L. Cuiyan, Z. Dongchun, Z. Xianyi, A survey of repetitive control, in: Proceedings of 2004 IEEE/RSJ International Conference on Intelligent Robots and Systems, 2004, pp. 1160-1166.

[30] R.W. Longman, Iterative learning control and repetitive control for engineering practice, International Journal of Control 73 (10) (2000) 930-954.

[31] G. Pinte, B. Stallaert, P. Sas, W. Desmet, J. Swevers, A novel design strategy for iterative learning and repetitive controllers of systems with a high modal density: theoretical background, Mechanical Systems and Signal Processing 24 (2) (2010) 432-443.

[32] J.S. Freudenberg, D.P. Looze, Right half plane poles and zeros and design trade-offs in feedback systems, IEEE Transactions on Automatic Control 30 (6) (1985) 555-565.

[33] G. Pipeleers, B. Demeulenaere, J. De Schutter, J. Swevers, Robust high-order repetitive control: optimal performance trade-offs, Automatica 44 (2008) 2628-2634. 